\title{
Dimensions of the ascending aorta in children and adolescents with repaired Tetralogy of Fallot obtained by cardiac magnetic resonance angiography
}

\author{
Matthias Grothoff $^{1} \cdot$ Meinhard Mende $^{2} \cdot$ Daniel Graefe $^{1} \cdot$ Ingo Daehnert $^{3}$ • \\ Martin Kostelka $^{4} \cdot$ Janine Hoffmann $^{5}$ - Patrick Freyhardt ${ }^{6} \cdot$ Lukas Lehmkuhl $^{1}$. \\ Matthias Gutberlet ${ }^{1}$ - Anne Mahler ${ }^{1,7}$
}

Received: 28 July 2015/ Accepted: 27 August 2015/Published online: 2 September 2015

(c) The Author(s) 2015. This article is published with open access at Springerlink.com

\begin{abstract}
Introduction Dilatation of the ascending aorta is a common finding in Tetralogy of fallot (TOF). We sought to provide aortic dimensions in children and adolescents after corrected TOF obtained by contrast-enhanced cardiacmagnetic-resonance angiography (CE-CMRA) that could serve as reference values.

Materials and methods We enrolled 101 children and adolescents (56 male) with a median age of 10.9 years. All patients underwent CE-CMRA imaging using a 3-dimensional spoiled gradient-echo-sequence. Aortic diameters were measured at the level of the aortic valve (AV), aortic sinus (AS), sino-tubular junction (STJ) and the ascending aorta (AA) and compared with normal values obtained
\end{abstract}

M. Gutberlet and A. Mahler should be both considered as senior authors.

Matthias Grothoff

grothoff@gmx.de

1 Department of Radiology, Heart Center, University of Leipzig, Struempellstr. 34, 04289 Leipzig, Germany

2 Clinical Trial Centre, University of Leipzig, Haertelstr. 16-18, 04107 Leipzig, Germany

3 Department of Pediatric Cardiology, Heart Center, University of Leipzig, Struempellstr. 34, 04289 Leipzig, Germany

4 Department of Cardiac Surgery, Heart Center, University of Leipzig, Struempellstr. 34, 04289 Leipzig, Germany

5 Department of Gynecology and Obstetrics, University of Leipzig, Liebigstr. 20a, 04103 Leipzig, Germany

6 Department of Radiology, Charité University Hospital, Augustenburger Platz 1, 13353 Berlin, Germany

7 Department of Intensive Care and Intermediate Care, University Hospital Aachen, Pauwelsstraße 30, 52074 Aachen, Germany from literature. Sex-specific aortic dimensions are given as percentile curves as well as $z$ scores. Furthermore CMR volumetric and functional parameters as well as clinical and anamnestic data were analyzed to identify parameters that are associated with aortic dilatation.

Results Diameters for aortic size for males were $3.6+16.6 * \mathrm{BSA}^{0.5}$ at the AV level, $7.0+19.5 * \mathrm{BSA}^{0.5}$ at the AS level, $7.0+14.4 * \mathrm{BSA}^{0.5}$ at the STJ level and $7.3+15.5 * \mathrm{BSA}^{0.5}$ at the AA level. Diameters for females were $5.8+14.1 * \mathrm{BSA}^{0.5}$ at the AV level, $7.2+17.6 * \mathrm{BSA}^{0.5}$ at the AS level, $5.2+15.4 * \mathrm{BSA}^{0.5}$ at the STJ level and $2.0+17.8^{*} \mathrm{BSA}^{0.5}$ at the AA level. All diameters in TOF patients were larger compared with normal values. The postoperative interval and age at examination were the only parameters associated with aortic size at all measured levels.

Conclusion We provide CE-CMRA data of aortic dimensions in children and adolescents after correction of TOF. Our data might be useful for an estimation of the "normal" aortic size in this patient cohort and can serve as a basis for future longitudinal studies adding prognostic data.

Keywords Tetralogy of Fallot - Aortic aneurysm . Magnetic resonance angiography $\cdot$ Reference values

\section{Introduction}

Tetralogy of Fallot (TOF) is the most common cyanotic congenital heart disease (CHD) [1]. Albeit TOF is defined by changes of the right ventricle and the pulmonary vessels, also dilatation of the aortic root and the ascending aorta can occur [2]. The pathomechanism of aortic dilatation in TOF and other cyanotic congenital heart disease 
associated with restricted pulmonary perfusion is unclear. The congenital anatomy, mostly prenatally manifested in form of right ventricular outflow obstruction or atresia and malalignment ventricular septal defect, which results in restricted flow to the pulmonary vessel and increased flow from both chamber to the aorta may play the major role. In such congenital heart disease with restricted pulmonary perfusion, the aortic dilatation is often detectable by fetal echocardiography. In addition, also genetic predisposition might contribute to aortic dilatation. It could, for instance, be shown that fibrillin-1 genetic mutations in TOF patients are associated with histologic abnormalities and larger aortic size [3-5].

Although rare, aortic dilatation can lead to the development of aneurysms and aortic dissection [6]. Therefore, early detection by close follow-up and timely intervention might help to improve the prognosis of fallot patients also with regard to aortic complications.

In literature, the prevalence of aortic root dilatation in TOF patients varies widely depending on the used imaging tool and the sizing criteria $[2,7]$. Echocardiography is the first line imaging modality in CHD and previous studies have established reference values for aortic size after correction of TOF [8]. Besides echocardiography also cardiac-magnetic resonance (CMR) is a routine follow-up tool in these patients. Typical elements of CMR protocols are steady state-free precession (SSFP) sequences for the assessment of biventricular morphology, size and function. Recently, age- and sex-specific pediatric reference values for ventricular volumes have been published [9]. In many centers including ours, also contrast-enhanced CMR angiography (CE-CMRA) is part of the follow-up imaging protocol. It is a robust tool to visualize the morphology of the great vessels and offers the possibility of multiple measurements at different positions in multiplanar reconstructions during post processing.

The aim of this study was to report diameters of the aortic root and the ascending aorta (AA) obtained by CECMRA in children and young adults after correction of TOF and compare them to normal values derived from literature. We furthermore sought to identify parameters that are associated with aortic dilatation.

\section{Materials and methods}

\section{Patient population}

This study consists of 101 consecutive patients younger than 21 years of age after surgical correction of TOF undergoing CMR for follow-up. All patients came for regular outpatient visits to our tertiary care center. None of our patients was diagnosed with Marfan syndrome, EhlersDanlos syndrome or any other connective tissue disorder. At the time of the CMR examination none of our patients had a right-to-left shunt or a major aortopulmonary collateral artery.

\section{CMR image acquisition}

All CMR examinations were performed on a 1.5 Tesla scanner (Gyroscan ACS-NT, Philips Healthcare, Best, The Netherlands) with a five-channel phased-array surface coil in supine position.

CE-CMRA images of the aorta were acquired using a 3-dimensional spoiled gradient-echo sequence in a coronary orientation with a repetition time of $2.9 \mathrm{~ms}$, echo time $1 \mathrm{~ms}$, flip angle $30^{\circ}$ and matrix of $480 \times 480 \times 48$. The field of view was adjusted to the patient size. Typical voxel size was $0.9 \times 0.9 \times 1.8 \mathrm{~mm}^{3}$, acquisition time $12 \mathrm{~s}$. Two CE-CMRA examinations were performed in each patient. The first acquisition was manually started when the contrast appeared in the pulmonary trunc, the second acquisition was started automatically immediately after the first sequence for visualizing the aorta. In children younger than 6 years a weight adapted dose of $0.2 \mathrm{mmol} / \mathrm{kg}$ bodyweight Gadopentetat-Dimeglumin was administered. In children older than 6 years with a body weight $<50 \mathrm{~kg}, 0.2 \mathrm{mmol} /$ $\mathrm{kg}$ bodyweight Gadubutrol was administered, in all others a standard dose of $15 \mathrm{mmol}$ Gadubutrol was used. Injection rate was $2 \mathrm{ml} / \mathrm{s}$ followed by a saline solution of $30 \mathrm{ml}$ with the same injection rate.

For the assessment of biventricular volumes and masses, a breath hold or free breathing navigator Cine-steady-statefree-precession (SSFP) sequence with retrospective ECGgating in an axial orientation and a slice thickness of $6 \mathrm{~mm}$ was used. Further sequence details have been published previously [10].

\section{CMR image analysis}

Measurements of aortic size were performed off-line on a separate workstation (Sectra PACS IDS5 11.4) by two blinded investigators with 4 (A.M.) and 15 (M.Gr.) years of experience in CMR. The aortic diameters were measured at four standardized levels, consisting of the aortic valve (AV), the aortic sinus (AS), the sino-tubular junction (STJ) and the AA. Each aortic segment was reconstructed in two double oblique planes as previously published [11]. First a parasagittal/paracoronal maximum intensity projection (MIP) of the aorta was reconstructed corresponding to a left anterior oblique view (Fig. 1). Then a second longitudinal plane perpendicular to the first plane was 
Fig. 1 Measurement of aortic diameters. Maximum intensity projection (MIP) in a left anterior oblique orientation (a). Longitudinal MIP perpendicular to the first (b). Cross-sectional plane perpendicular to both longitudinal views at the level of the sino-tubular junction (c)
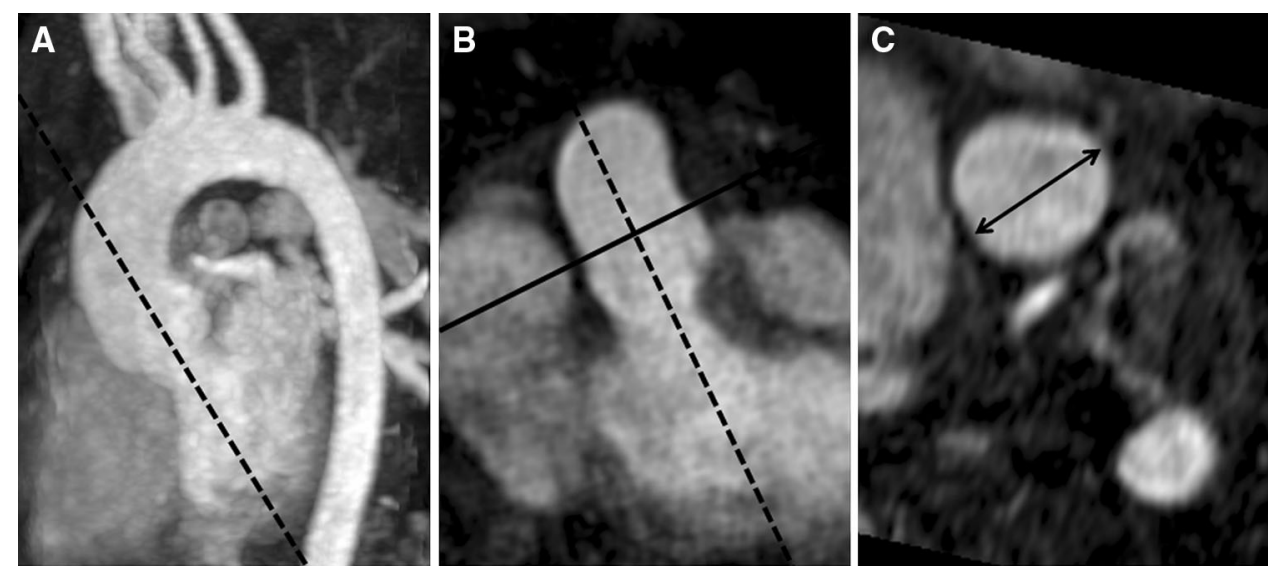

reconstructed. At last a third cross-sectional view perpendicular to both longitudinal views was angulated. At the level of the AS, the STJ and the AA the widest diameters were measured [12]. The aortic valve was measured at the level of the most basal attachment points of the three cusps to the aortic wall. Here two diameters perpendicular to each other were measured and the mean value was calculated.

Ventricular volumes were obtained by Simpson's rule according to the protocol of the German Competence Network of Congenital heart disease [13]. All parameters were related to body surface area (BSA) and shown as indices. We determined left ventricular (LV) and right ventricular (RV) end-diastolic volume index (EDVI), endsystolic volume index (ESVI) and stroke-volume index (SVI). Furthermore, we calculated biventricular ventricular ejection fractions $(\mathrm{EF})$.

\section{Anamnestic and clinical data}

The parameters age at surgical correction of TOF, age at CMR examination, postoperative interval, type of correction and number of previous palliative procedures were taken from patient records. New York Heart Association (NYHA) functional class as determined by a standardized questionnaire, the degree of aortic insufficiency (AI) measured by echocardiography according to the guidelines of the European Society of Cardiology and non-invasive arterial blood pressure (given in percentiles) were obtained during the clinical routine follow-up examination at the day of the CMR examination [14].

\section{Comparison with normal values}

Normative data were derived from the literature [11]. Aortic diameters could be compared at three corresponding levels (AS, STJ and AA). The equations for the percentiles of aortic diameters given in the cited study were used to calculate the predicted aortic diameters for the Fallot patients in the present study.

\section{Statistical analysis}

For statistical analysis IBM SPSS Statistics software package 20.0 (Chicago, IL, USA) was used. Intra- and interrater coefficients of variation $(\mathrm{CV})$ were estimated by means of ANOVA models with repeated measurements.

Checking the functional dependency, we found that aortic diameters fitted best to square root of BSA. Nonsignificant equivalence tests between girls and boys indicated that curves should be fitted separately for both sexes.

To calculate reference percentile curves the GAMLSS methodology [15] was used. It allows fitting several probability distributions with covariate dependent scale and shape parameters. Square root of BSA was chosen as appropriate covariate. Minimal global deviance as well as relatively smoothed curves and good agreement of estimated and observed quantiles were criteria for the choice of adequate model function. Skew exponential power family was applied four times, $t$ family two and skew $t 3$ family and Box-Cox $t$ family one time each.

Aortic diameters of our TOF patients were compared with previously published normal values using paired $t$ testing [11]. Furthermore our values were classified in a $z$ value coordinate system basing on the previously published normal values.

Looking for covariates associated with aortic diameters, we proceeded in three steps in analogy to Sauerbrei and Schumacher [16]. First we performed bagging procedures based on 15 CMR volumetric and functional parameters as well as clinical and anamnestic parameters, i.e., each 100 linear models from bootstrap samples were fitted using the best subset criterion based on the Akaike Information Criterion. In step two we included only such variables that 
were in more than $50 \%$ models into a linear model. In step three potentially a non-significant variable had to be excluded to get the final model.

\section{Results}

The study cohort consisted of 56 male and 45 female patients with a median age of 10.9 years (range 10.1-20.2 years). The median BSA calculated from the Mosteller formula was $1.2 \mathrm{~m}^{2}$ (range $0.6-2.2 \mathrm{~m}^{2}$ ) with a median height of $133 \mathrm{~cm}$ (range 92-182 cm) and a median weight of $28.5 \mathrm{~kg}$ (range 12.0-94.8 kg) [17]. The median body mass index was $16.0 \mathrm{~kg} / \mathrm{m}^{2}$ (range $11.8-32.7 \mathrm{~kg} / \mathrm{m}^{2}$ ).

Parameters of the control cohort [11] were almost identical (Table 1).

The median and range of the measured aortic segments are given in Table 2. Mann-Whitney $U$ test of the indexed aortic diameters revealed no difference between male and female patients. However, because equivalence testing procedure failed to show equivalence, sex-specific values are given. Percentile equations are shown in Table 3. Percentile curves are shown in Figs. 2 and 3.

Compared with normal values the Fallot group showed larger aortic diameters at all comparable levels with significant differences between the predicted [11] and measured diameters (Fig. 4). The mean differences and standard deviations were: $5.9 \pm 2.9 \mathrm{~mm}$ at the AS level, $5.2 \pm 2.9 \mathrm{~mm}$ at the STJ level and $4.8 \pm 3.1 \mathrm{~mm}$ at the AA level ( $p<0.001$ for all).

For readers convenience we provide online electronic calculators of $z$ scores when compared with the Fallot group (http://tof.pedz.de) and when compared to normal aortas (http://mri.pedz.de).

The coefficients of variations for intraobserver variability were: $8 \%$ at the AA level, $6.3 \%$ at the AS level, $5.8 \%$ at the STJ level and $5.9 \%$ at the AA level. The coefficients of variations for interobserver variability were: $6.5 \%$ at the AA level, $6.1 \%$ at the AS level, $6.0 \%$ at the STJ level and $5.8 \%$ at the AA level.

In CMR planimetry median LV-EDVI was $79.6 \mathrm{ml} / \mathrm{m}^{2}$ (range $40-172 \mathrm{ml} / \mathrm{m}^{2}$ ), LV-ESVI was $34.5 \mathrm{ml} / \mathrm{m}^{2}$ (range

Table 1 Patient parameters

\begin{tabular}{lll}
\hline & Fallot patients & Controls derived from [11] \\
\hline Number of patients & 101 & 53 \\
Male:female $(\%)$ & $55: 45$ & $57: 43$ \\
Age (year) & $10.9(2-20)$ & $9(2-20)$ \\
Body surface area $\left(\mathrm{m}^{2}\right)$ & $1.02(0.55-2.1)$ & $1.05(0.52-1.9)$ \\
Height $(\mathrm{cm})$ & $133(92-182)$ & $131(81-184)$ \\
Weight $(\mathrm{kg})$ & $28.5(12-95)$ & $30(12-75)$ \\
\hline
\end{tabular}

Continuous data are presented as median and range

Table 2 Sex-specific diameters of the aorta

\begin{tabular}{|c|c|c|c|c|}
\hline & \multicolumn{2}{|l|}{ Males } & \multicolumn{2}{|l|}{ Females } \\
\hline & Median & Range & Median & Range \\
\hline Aortic valve $\left(\mathrm{mm} / \mathrm{m}^{2}\right)$ & 18.3 & $11.4-31.2$ & 17.7 & $11.5-27.9$ \\
\hline Aortic sinus $\left(\mathrm{mm} / \mathrm{m}^{2}\right)$ & 22.6 & $14.0-44.1$ & 22.3 & $14.8-40.3$ \\
\hline Sino-tubular junction $\left(\mathrm{mm} / \mathrm{m}^{2}\right)$ & 19.9 & $11.5-38.2$ & 18.7 & $14.0-32.2$ \\
\hline Ascending aorta $\left(\mathrm{mm} / \mathrm{m}^{2}\right)$ & 20.3 & $10.4-42.5$ & 19.3 & $12.8-30.0$ \\
\hline
\end{tabular}

Table 3 Sex-specific functions for aortic diameters

\begin{tabular}{lllllll}
\hline & \multicolumn{2}{l}{ Males } & & \multicolumn{2}{l}{ Females } \\
\cline { 2 - 3 } & Predicted diameter & & SD of residuals $(\mathrm{mm})$ & & Predicted diameter & SD of residuals $(\mathrm{mm})$ \\
\hline Aortic valve & $3.6+16.6 * \mathrm{BSA}^{0.5}$ & 2.4 & & $5.8+14.1 * \mathrm{BSA}^{0.5}$ & 2.3 \\
Aortic sinus & $7.0+19.5 * \mathrm{BSA}^{0.5}$ & 3.5 & & & $7.2+17.6 * \mathrm{BSA}^{0.5}$ & 3.2 \\
Sino-tubular junction & $7.0+14.4 * \mathrm{BSA}^{0.5}$ & 4.3 & & $5.2+15.4 * \mathrm{BSA}^{0.5}$ & 3.5 \\
Ascending aorta & $7.3+15.5 * \mathrm{BSA}^{0.5}$ & 4.0 & & $2.0+17.8 * \mathrm{BSA}^{0.5}$ & 3.5 &
\end{tabular}



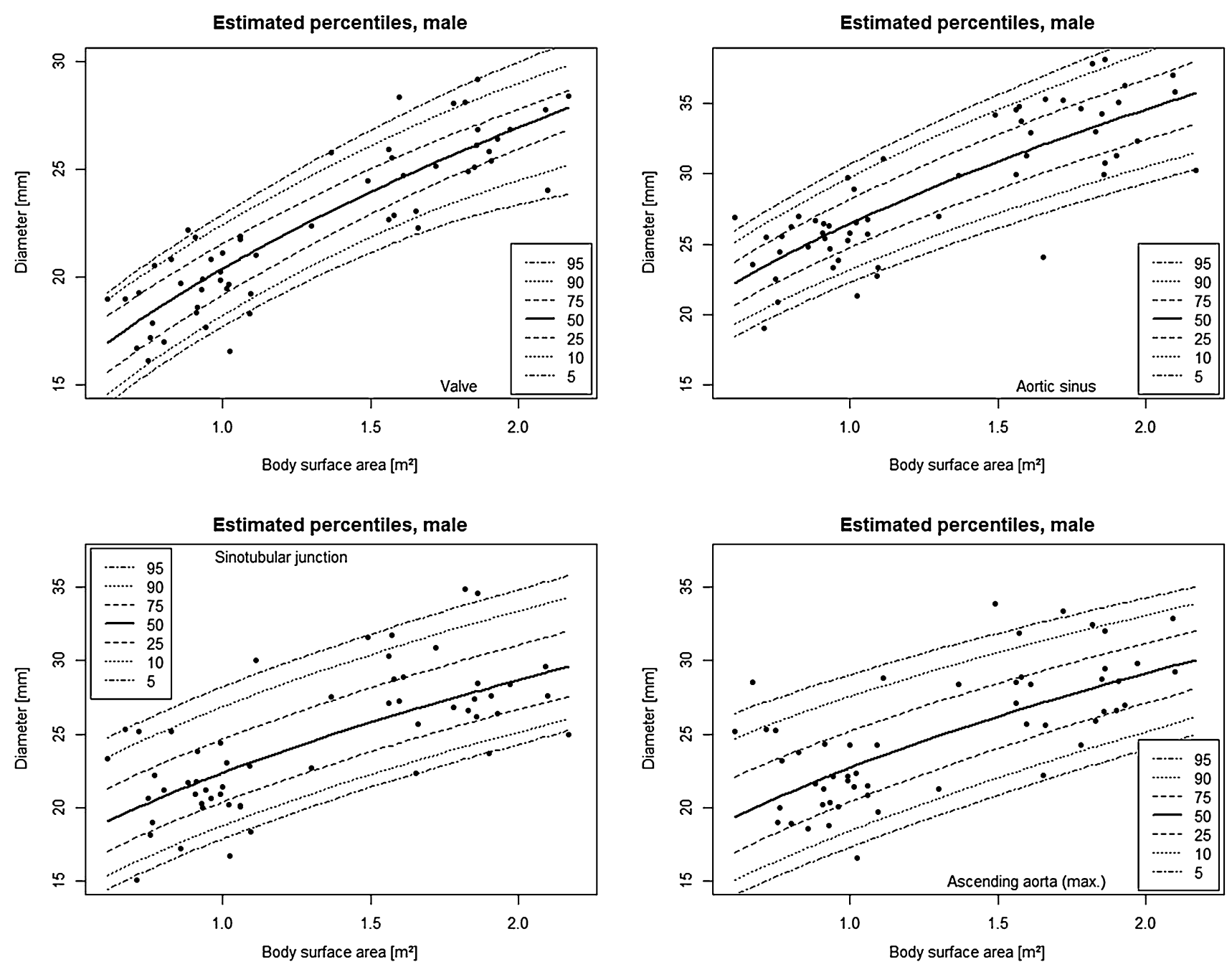

Fig. 2 Percentile curves for aortic diameters after correction of TOF in male children and young adults

15-92 $\mathrm{ml} / \mathrm{m}^{2}$ ), LV-SVI was $46.0 \mathrm{ml} / \mathrm{m}^{2}$ (range $21-120 \mathrm{ml} /$ $\mathrm{m}^{2}$ ) and LV-EF was $56.0 \%$ (range 28-79 \%). Median RVEDVI was $110.6 \mathrm{ml} / \mathrm{m}^{2}$ (range $53-215 \mathrm{ml} / \mathrm{m}^{2}$, RV-ESVI was $57.0 \mathrm{ml} / \mathrm{m}^{2}$ (range $18-173 \mathrm{ml} / \mathrm{m}^{2}$ ), RV-SVI was $53.1 \mathrm{ml} / \mathrm{m}^{2}\left(20-176 \mathrm{ml} / \mathrm{m}^{2}\right)$ and RV-EF was $47.9 \%$ (range $25-74 \mathrm{ml} / \mathrm{m}^{2}$ ).

No significant associations were found between these parameters and aortic dimensions at the four measured levels.

Median age at surgical correction was 0.8 years (range 0.1-11.9 years), median postoperative interval was 9.7 years (range 2.0-19.1 years). A transannular approach was used in 74/101 patients, a subvalvular patching in $18 / 101$ patients, a conduit was used in 7/101 patients. In two patients data regarding the used surgical method were inconclusive or missing. In 16/101 patients palliative procedures had been performed, 14 patients had one palliation and one patient had two and three palliations, respectively. Pulmonary atresia was corrected in $6 / 101$ patients.

The postoperative interval and age at CMR examination were the only parameters that were associated with aortic size at the four measured levels $(p<0.001$ for all), meaning patients with a longer interval and a higher age had larger aortic dimensions. These two parameters were highly interdependent as the majority of patients were corrected within the first year of life. Age at corrective surgery, type of correction as well as palliations were not associated with aortic size. Also the presence of a right aortic arch, which was found in 25 patients (25\%), and pulmonary atresia were not associated with aortic size.

NYHA functional class I was found in 74/101 patients, class II in $18 / 101$ patients and class III in $1 / 101$ patients. In eight patients data were missing. NYHA functional class was not associated with aortic size. 
Estimated percentiles, female

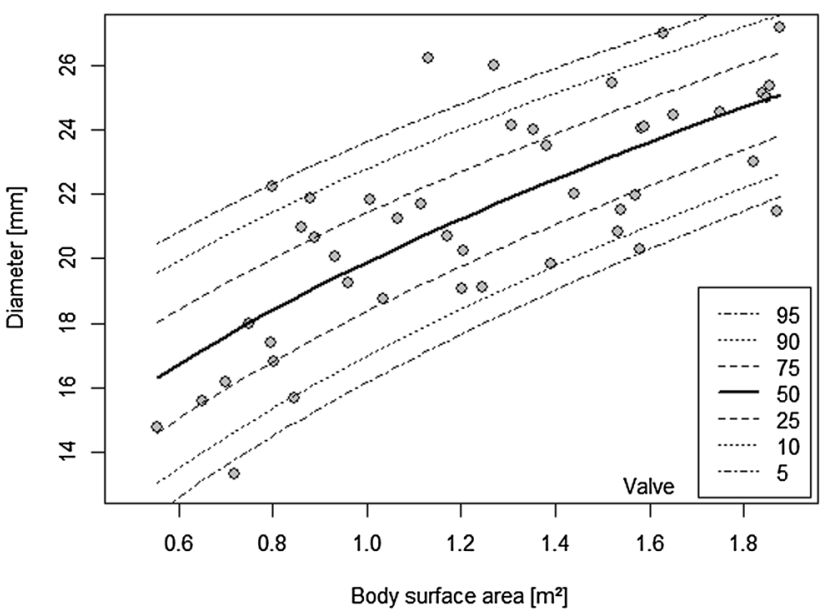

Estimated percentiles, female

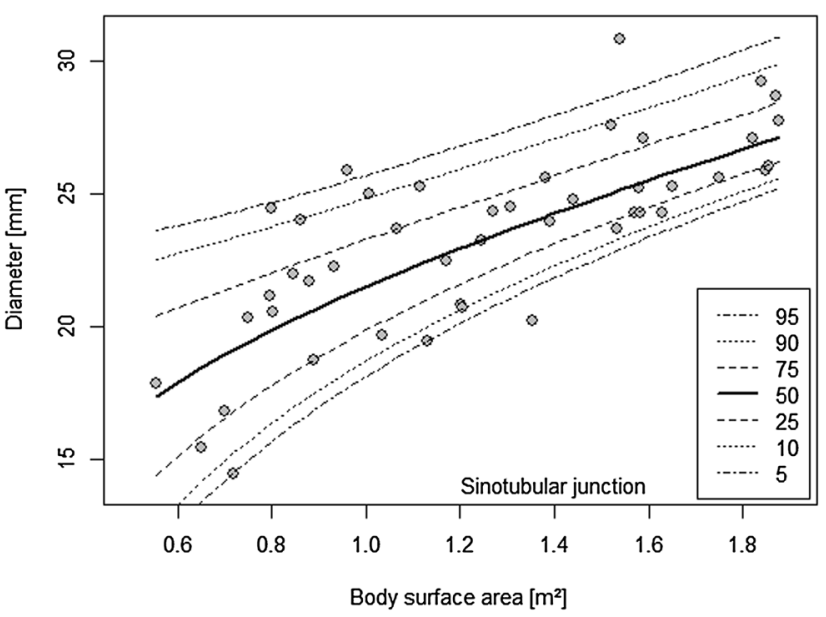

Estimated percentiles, female

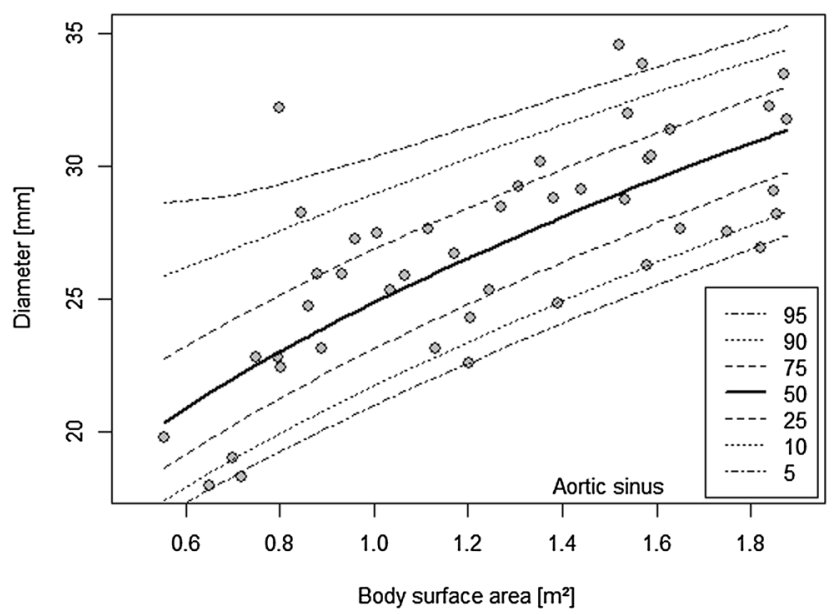

Estimated percentiles, female

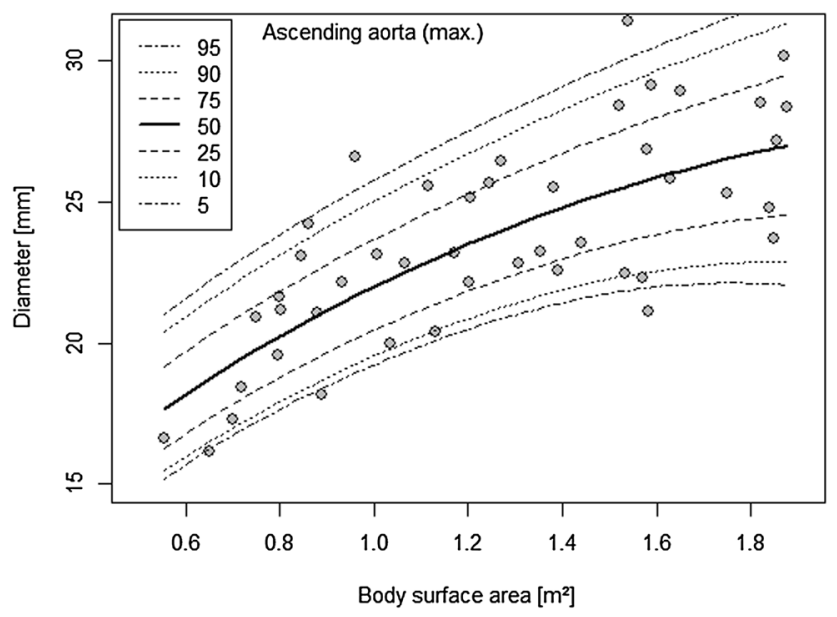

Fig. 3 Percentile curves for aortic diameters after correction of TOF in female children and young adults
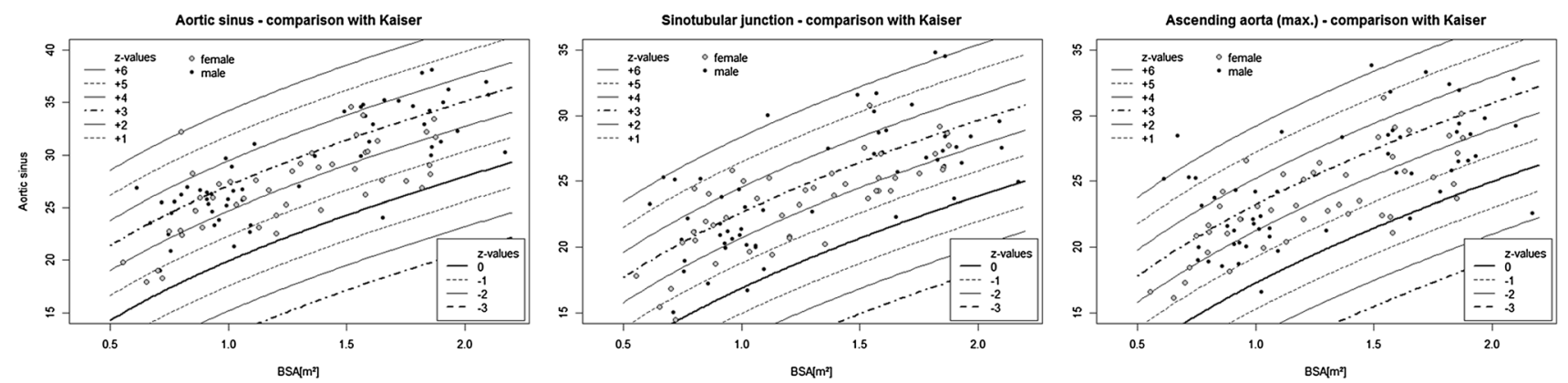

Fig. $4 z$ values of aortic diameters in TOF compared with normal aortic values according to [11]

Echocardiography showed competent aortic valves in $75 / 101$ patients, a mild AI in 20/101 patients and a moderate $\mathrm{AI}$ in 2/101 patients. In four patients data were inconclusive or missing. Median systolic blood pressure was below the 90th percentile, median diastolic blood pressure was on the 50th percentile. AI and blood pressure were not associated with aortic size.

\section{Discussion}

It is well-known that TOF can be associated with a clinically relevant dilatation of the aorta and echocardiography is the first line imaging tool for measuring aortic dimensions. In addition to echocardiography also CMR is an inherent part of the follow-up work flow in congenital heart 
disease and recently a number of CMR derived normative date have been published [18-21].

Our study adds CMR data of the aortic size in children and adolescents after correction of TOF that could serve as reference values.

Besides the unique capabilities of CMR in imaging morphologic and functional changes of the right heart after correction of TOF also imaging of the great vessels is feasible without restrictions from patient constitution and the presence of an adequate acoustic window. In most centers the CMR protocol includes the administration of a gadolinium based contrast-medium for CE-CMRA and late gadolinium enhancement (LGE) imaging. The CE-CMRA sequence is primarily used for visualization of the right ventricular outflow tract and the pulmonary arteries. Yet the short acquisition time also allows imaging of the entire thoracic aorta with optimal timing using the same contrast bolus.

The acquisition of a 3-dimensional CE-CMRA dataset offers some advantages over other CMR techniques like 2-dimensional spin-echo, phase contrast or steady statefree precession imaging. In a 3-dimensional CE-CMRA dataset it is possible to create multiplanar reconstructions and to perform multiple measurements. Once the source data is acquired all measurements can be performed offline in any position and there is no need for a correct crosssectional angulation pre imaging. In our study we provide normal values in four positions of the aortic root and the ascending aorta. As known from echocardiography studies, enlargement in postoperative TOF is not limited to a single section of the aorta but can effect multiple segments [22, 23]. Thus a gapless acquisition might be better than the acquisition of single slices. Moreover CE-CMRA sequences are based on differences in $T 1$ relaxation times and not on inflow effects what gives them a high signal to noise ratio and makes them insensitive to flow artifacts.

The robustness of this method is underlined by a low intra- and interobserver variability of our data. The reliability of measurements is particularly important in CHD as therapeutic decisions are often based on the time course of morphological and functional changes. Therefore the capability to detect minor changes and tendencies is crucial. Interobserver variability was highest at the level of the $\mathrm{AV}$ and the AS and lowest at the level of the AA. This is a common finding in CE-CMRA measurements of the aorta as the 3-dimensional dataset is acquired over multiple cardiac cycles without electrocardiographic triggering and irrespective of vessel wall motion. Therefore the contours of the highly pulsatile proximal vessel sections show more blurring than the more distal sections [11, 24].

Although growth of cardiovascular structures is an allometric process, indexing cardiac parameters to BSA is common practice not only in adult but also in pediatric cardiology [25]. According to previous studies our data show that growth of the aorta is best described by a linear relationship between aortic diameters and the square root of the BSA $[11,26]$. In addition we provide sex-specific values. Previous studies found bigger valve diameters in boys at all ages, even before adolescence [27, 28]. Moreover onset and time course of growth is sex- specific. Pooled values would have underestimated male and overestimated female aortic diameters.

Aortic diameters of our TOF cohort are significantly larger than the normal aortic values published by Kaiser et al. [11]. Numerous previous echocardiography studies have reported enlarged aortas in TOF, yet the prevalence of aortic dilatation varies widely depending on definition and cut-off values [2, 7, 29]. In a large multicenter study an aortic root diameter of $\geq 40 \mathrm{~mm}$ was found in $29 \%$ of adult TOF patients [22]. However, there is only little data on aortic size measured with CMR. Recently Kutty et al. published CMR data of AA size in a large group of TOF patients [18]. Compared with our data AA diameters were slightly higher in the cited study, which might be explained by methodological differences in AA assessment. Kutty et al. used phase contrast imaging for measuring the AA, meaning that the aortic wall was included into the diameters. In CE-CMRA the lumen of the aorta appears hyperintense caused by the gadolinium based shortening of $T 1$ relaxation times. The surrounding tissue including the vessel walls is hypointense. Measurements in CE-CMRA therefore exclude vessel walls and can rather be compared to luminography in conventional angiography. Moreover age at repair was higher in the cited study. The majority of our patients underwent corrective surgery within the first year of life and might therefore be considered a more 'contemporary' TOF cohort of children and adolescents as an early repair is standard in most centers nowadays.

Yet it is unclear whether an early repair can reduce, delay or even prevent aortic dilatation in TOF. Though it is assumed that increased aortic flow attributable to right-toleft shunting might lead to high wall shear stress also intrinsic alterations of the aortic wall seem to play a role in the pathophysiology [30]. Actually our data did not show a significant association between the age at repair and aortic size. The only parameters associated with an increased diameter at all measured levels were the postoperative interval and the age at CMR examination. As our group was quite homogeneous with regard to an early repair, these two parameters were highly interdependent and should be interpreted as a continuous growth of aortic dimensions over time. Our results support the thesis of an intrinsic aortopathy rather than the thesis of preoperative hemodynamic alterations.

Male sex was associated with non-significant larger aortas. In contradiction to previous studies neither right 
aortic arches nor increased LV volumes were associated with aortic dilatation [2, 4]. Large prospective longitudinal multicenter studies are necessary to clearly identify independent factors of aortic dilatation in TOF.

The natural course of the left-sided affection of TOF is discussed controversially [23, 31]. The mild degree of aortic dilation in repaired TOF patients as shown by our results reflects our clinical experience. Severe complications due to this dilation are rare and there only exist sporadic reports of aortic dissection or even rupture. Yet in future decades the number of adult and elderly patients with corrected TOF will increase and atherosclerosis may put these dilated aortas at risk for dissection. In addition a dilated aorta could result in a compression of the right pulmonary artery with unequal pulmonary perfusion and in the worst case leading to left-sided pulmonary hypertension with the need of a proximal aortic replacement [32]. Moreover, aortic dilatation at the level of the AV and the AS can lead to AI. In the present study AI was obtained by echocardiography and there were only a small number of young patients showing mild to moderate AI. This might be the reason for the absence of a correlation between aortic size and aortic regurgitation. Further studies should use CMR phase contrast flow measurement for an absolute quantification of $\mathrm{AI}$ and a more detailed analysis.

Our results show the distribution of aortic dimensions in patients with corrected TOF. This data may be useful for an estimation of the 'normal' aortic size in this patient cohort.

Special guidelines regarding the optimal time of replacement of the ascending aorta in patients with connective tissue diseases (e.g. Marfan syndrome) as well as normal variants (e.g. bicuspid aortic valve) have been published [12]. No such cut-off values exist for patients with corrected TOF. Although we cannot provide such data with our current approach this study might be a basis for future longitudinal studies using CE-CMRA.

\section{Limitations}

The smallest BSA in this study was $0.52 \mathrm{~m}^{2}$. Therefore, we cannot provide reference values for neonates and very young infants. However, this group of patients is not anyway routinely examined by CMR in follow-up examinations in TOF as the procedure is time consuming and needs sedation.

Although our study population consisted of a relatively large number of TOF patients, it is a cross-sectional study with the given limitations in identifying parameters that are associated with aortic growth. Larger longitudinal studies might give further insight into the time course and risk factors of aortic dilatation in TOF.
At last this study has the typical limitations of a retrospective approach. Some data regarding the type of surgery and palliative procedures were missing in our analysis.

\section{Compliance with ethical standards}

Conflict of interest The authors declare that they have no conflict of interest.

Ethical standard This study has been approved by the local ethics committee and has been performed in accordance with the ethical standards of the Declaration of Helsinki and its later amendments. All patients or legal guardians gave their informed consent for the scientific use of their data prior to the inclusion in the study.

Open Access This article is distributed under the terms of the Creative Commons Attribution 4.0 International License (http://crea tivecommons.org/licenses/by/4.0/), which permits unrestricted use, distribution, and reproduction in any medium, provided you give appropriate credit to the original author(s) and the source, provide a link to the Creative Commons license, and indicate if changes were made.

\section{References}

1. Murphy JG, Gersh BJ, Mair DD et al (1993) Long-term outcome in patients undergoing surgical repair of tetralogy of Fallot. N Engl J Med 329:593-599

2. Niwa K, Siu SC, Webb GD, Gatzoulis MA (2002) Progressive aortic root dilatation in adults late after repair of tetralogy of Fallot. Circulation 106:1374-1378

3. Seki M, Kurishima C, Kawasaki H, Masutani S, Senzaki H (2012) Aortic stiffness and aortic dilation in infants and children with tetralogy of Fallot before corrective surgery: evidence for intrinsically abnormal aortic mechanical property. Eur J Cardiothorac Surg 41:277-282

4. Tan JL, Davlouros PA, McCarthy KP, Gatzoulis MA, Ho SY (2005) Intrinsic histological abnormalities of aortic root and ascending aorta in tetralogy of Fallot: evidence of causative mechanism for aortic dilatation and aortopathy. Circulation 112:961-968

5. Chowdhury UK, Mishra AK, Ray R, Kalaivani M, Reddy SM, Venugopal P (2008) Histopathologic changes in ascending aorta and risk factors related to histopathologic conditions and aortic dilatation in patients with tetralogy of Fallot. J Thorac Cardiovasc Surg 135:69-77

6. Rathi VK, Doyle M, Williams RB, Yamrozik J, Shannon RP, Biederman RWW (2005) Massive aortic aneurysm and dissection in repaired tetralogy of Fallot; diagnosis by cardiovascular magnetic resonance imaging. Int J Cardiol 101:169-170

7. Chong W-Y, Wong WHS, Chiu CSW, Cheung Y-F (2006) Aortic root dilation and aortic elastic properties in children after repair of tetralogy of Fallot. Am J Cardiol 97:905-909

8. Gautier M, Detaint D, Fermanian C et al (2010) Nomograms for aortic root diameters in children using two-dimensional echocardiography. Am J Cardiol 105:888-894

9. Sarikouch S, Peters B, Gutberlet M et al (2010) Sex-specific pediatric percentiles for ventricular size and mass as reference values for cardiac MRI: assessment by steady-state free-precession and phase-contrast MRI flow. Circ Cardiovasc Imaging 3:65-76

10. Grothoff M, Hoffmann J, Lehmkuhl L et al (2011) Time course of right ventricular functional parameters after surgical correction of 
tetralogy of Fallot determined by cardiac magnetic resonance. Clin Res Cardiol 100:343-350

11. Kaiser T, Kellenberger CJ, Albisetti M, Bergsträsser E, Valsangiacomo Buechel ER (2008) Normal values for aortic diameters in children and adolescents - assessment in vivo by contrast-enhanced CMR-angiography. J Cardiovasc Magn Reson 10:56

12. Hiratzka LF, Bakris GL, Beckman JA et al (2010) ACCF/AHA/ AATS/ACR/ASA/SCA/SCAI/SIR/STS/SVM guidelines for the diagnosis and management of patients with Thoracic Aortic Disease: a report of the American College of Cardiology Foundation/American Heart Association Task Force on Practice Guidelines, American Association for Thoracic Surgery, American College of Radiology, American Stroke Association, Society of Cardiovascular Anesthesiologists, Society for Cardiovascular Angiography and Interventions, Society of Interventional Radiology, Society of Thoracic Surgeons, and Society for Vascular Medicine. Circulation 121:e266-e369

13. Beerbaum P, Barth P, Kropf S et al (2009) Cardiac function by MRI in congenital heart disease: impact of consensus training on interinstitutional variance. J Magn Reson Imaging 30:956-966

14. Lancellotti P, Tribouilloy C, Hagendorff A et al (2013) Recommendations for the echocardiographic assessment of native valvular regurgitation: an executive summary from the European Association of Cardiovascular Imaging. Eur Heart J Cardiovasc Imaging 14:611-644

15. Rigby RA, Stasinopoulos DM (2005) Generalized additive models for location, scale and shape. J R Stat Soc Ser C Appl Stat 54:507-554

16. Sauerbrei W, Schumacher M (1992) A bootstrap resampling procedure for model building: application to the Cox regression model. Stat Med 11:2093-2109

17. Mosteller RD (1987) Simplified calculation of body-surface area. N Engl J Med 317:1098

18. Kutty S, Kuehne T, Gribben P et al (2012) Ascending aortic and main pulmonary artery areas derived from cardiovascular magnetic resonance as reference values for normal subjects and repaired tetralogy of Fallot. Circ Cardiovasc Imaging 5:644-651

19. Sarikouch S, Koerperich H, Dubowy KO et al (2011) Impact of gender and age on cardiovascular function late after repair of tetralogy of Fallot: percentiles based on cardiac magnetic resonance. Circ Cardiovasc Imaging 4:703-711

20. Grothoff M, Spors B, Abdul-Khaliq H et al (2006) Pulmonary regurgitation is a powerful factor influencing QRS duration in patients after surgical repair of tetralogy of Fallot. A magnetic resonance imaging (MRI) study. Clin Res Cardiol 95:643-649

21. Preim U, Hoffmann J, Lehmkuhl L et al (2013) Systemic right ventricles rarely show myocardial scars in cardiac magnetic resonance delayed-enhancement imaging. Clin Res Cardiol 102:337-344

22. Mongeon F-P, Gurvitz MZ, Broberg CS et al (2013) Aortic root dilatation in adults with surgically repaired tetralogy of fallot: a multicenter cross-sectional study. Circulation 127:172-179

23. Kay WA, Cook SC, Daniels CJ (2013) Evaluation by MRA of aortic dilation late after repair of tetralogy of Fallot. Int J Cardiol 167:2922-2927

24. Russo V, Renzulli M, La Palombara C, Fattori R (2006) Congenital diseases of the thoracic aorta. Role of MRI and MRA. Eur Radiol 16:676-684

25. Gutgesell HP, Rembold CM (1990) Growth of the human heart relative to body surface area. Am J Cardiol 65:662-668

26. Sluysmans T, Colan SD (2005) Theoretical and empirical derivation of cardiovascular allometric relationships in children. J Appl Physiol (1985) 99:445-457

27. Scholz DG, Kitzman DW, Hagen PT, Ilstrup DM, Edwards WD (1988) Age-related changes in normal human hearts during the first 10 decades of life. Part I (Growth): a quantitative anatomic study of 200 specimens from subjects from birth to 19 years old. Mayo Clin Proc 63:126-136

28. Zilberman MV, Khoury PR, Kimball RT (2005) Two-dimensional echocardiographic valve measurements in healthy children: gender-specific differences. Pediatr Cardiol 26:356-360

29. Apitz C, Webb GD, Redington AN (2009) Tetralogy of Fallot. Lancet 374:1462-1471

30. Niwa K (2005) Aortic root dilatation in tetralogy of Fallot longterm after repair -histology of the aorta in tetralogy of Fallot: evidence of intrinsic aortopathy. Int J Cardiol 103:117-119

31. François K, Creytens D, De Groote K et al (2014) Analysis of the aortic root in patients with tetralogy of Fallot undergoing early repair: form follows function. J Thorac Cardiovasc Surg 148:1555-1559

32. Kunihara T, Aicher D, Asano M et al (2014) Risk factors for prophylactic proximal aortic replacement in the current era. Clin Res Cardiol 103:431-440 